New Methods

\title{
Adventitial Inversion with Graft Telescopic Insertion for Distal Anastomosis in Acute Type A Aortic Dissection
}

\author{
Bartosz Rylski, MD, ${ }^{1}$ Matthias Siepe, MD, ${ }^{1}$ Philipp Blanke, $\mathrm{MD},{ }^{2}$ Wulf Euringer, MD, ${ }^{2}$
} Joachim Schoellhorn, $\mathrm{MD},{ }^{1}$ and Friedhelm Beyersdorf, $\mathrm{MD}, \mathrm{PhD}^{1}$

\begin{abstract}
Purpose: One current method of anastomosis in aortic dissection type $A$ is the adventitial inversion technique. To improve hemostasis at the anastomotic site, we have developed a novel technique for distal anastomosis involving adventitial inversion employing graft telescopic insertion.

Methods: The adventitia was inverted into the aortic lumen and the anastomosis with a Dacron tube-graft was made in a telescopic method, covering the inverted adventitia.

Results: Five patients have undergone emergency ascending aortic replacement for aortic dissection by one surgeon using this technique. There have been no reoperations for bleeding or false aneurysm.

Conclusion: Complete coverage of the inverted adventitia eliminated the potential risk of thrombus formation. Graft telescopic insertion lead to complete hemostasis.
\end{abstract}

Keywords: aortic dissection, aortic surgery, anastomosis on the aorta

\section{Introduction}

An aortic wall affected by dissection is vulnerable, necessitating a particularly secure anastomosis. Although various anastomotic options have been described for reconstructive prosthetic techniques on the aorta, suboptimal adhesion of dissected aortic layers and hemostasis at anastomotic sites remain difficult to achieve. One current method is the adventitial inversion technique, firstly reported by Floten et al. ${ }^{1)}$ Recent studies confirmed this technique's feasibility and safeness, demonstrating that it

${ }^{1}$ University Cardiovascular Center Freiburg-Bad Krozingen, Freiburg, Germany

${ }^{2}$ Department of Diagnostic Radiology, University Hospital Freiburg, Freiburg, Germany

Received: October 11, 2011; Accepted: October 27, 2011

Corresponding author: Bartosz Rylski, MD. University Cardiovascular Center Freiburg-Bad Krozingen, Hugstetter Str. 55, 79106 Freiburg, Germany

Email: bartosz.rylski@uniklinik-freiburg.de

(C)2012 The Editorial Committee of Annals of Thoracic and

Cardiovascular Surgery. All rights reserved. facilitates thrombotic closure of the false lumen more effectively than other methods. ${ }^{2,3)}$ However, the intraluminal adventitia supposedly carries the risk of thrombus formation with subsequent embolization, which has been shown in experimental studies in small-caliber anastomoses. ${ }^{4)}$ To prevent this serious complication and improve hemostasis at the anastomotic site, we have developed a novel technique involving adventitial inversion employing, previously described by us, graft telescopic insertion. $^{5)}$

\section{Patients and Methods}

Aortic anastomosis was performed using the method described below. The axillary artery was cannulated, and we opted to employ antegrade cerebral perfusion with the brachiocephalic trunk occluded. After initiating retrograde blood cardioplegia, the diseased ascending aorta was resected, so that the adventitial margin was trimmed $1 \mathrm{~cm}$ longer than the level of the intimal edge. To prevent any distortion of innominate flow, distal aortic resection was carried out at about a 5-mm distance from the 

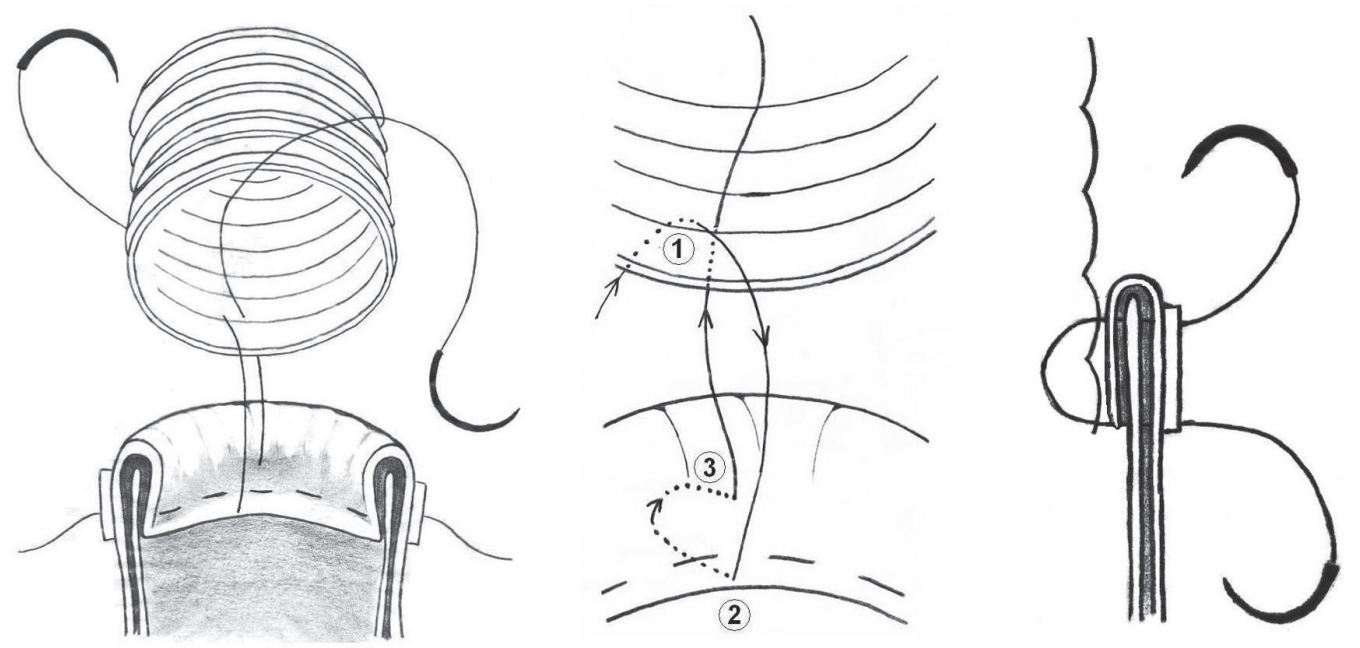

Fig. 1 A technique of adventitial inversion and telescopic graft insertion for acute type A aortic dissection. (A) The adventitia was trimmed $1 \mathrm{~cm}$ longer than the level of intimal edge and inverted into the aortic lumen using mattress sutures reinforced with an extraluminal circumferential felt strip. The anastomosis with a Dacron tube-graft was made using a continuous suture, (B1) the needle of which went through the graft (out-in), (B2) through the aorta (in-out), (B3) and again through the aorta (out-in). (C) About $5 \mathrm{~mm}$ of the graft was inserted into the aorta, completely covering the inverted adventitia.

innominate origin. The redundant adventitia was inverted into the aortic lumen using 4-0 polypropylene (Johnson \& Johnson Intl, St-Stevens Woluwe, Belgium) mattress sutures reinforced with an extraluminal circumferential felt strip. The anastomosis with a Dacron (Vascutek, Inchinnan, Scotland) tube-graft of appropriate size was made using a continuous 3-0 suture, the needle of which went through the graft (out-in), through the aorta (in-out), and again through the aorta (out-in) to insert 5-7 $\mathrm{mm}$ of the graft into the aorta, completely covering the inverted adventitia. At the end of the anastomosis, the running suture and the suture for the external felt were carefully retightened and knotted to prevent an anastomotic stenosis (Figs. 1 and 2). We used this technique for distal anastomosis in the supracoronary replacement of the ascending aorta.

\section{Results}

Five patients have undergone emergency ascending aortic replacement for aortic dissection by one surgeon using this technique. There have been no reoperations for bleeding or false aneurysm. One patient died on the intensive care unit due to acute respiratory distress syndrome. Our results are limited by the small sample size.

\section{Discussion}

One of the current anastomotic options for reconstructive prosthetic therapy on the aorta for acute type A dissection is the adventitial inversion technique. Since this method's initial introduction by Floten et al.," a few clinical studies have confirmed that it is a feasible and safe technique providing successful exclusion of the false lumen from antegrade flow at the anastomotic level. ${ }^{2,3)}$ However, there are some experimental reports suggesting that, due to the extrinsic coagulation pathway via exposed adventitial collagen and tissue factor, inverted adventitia may elicit thrombus formation with subsequent embolization, ${ }^{4)}$ although the thrombogenicity of inverted adventitia has not been tested clinically to our knowledge. Wishing to address this technique's advantages and its potential complications, we integrated this method with our method of telescopic graft insertion. ${ }^{5)}$ This resulted in complete coverage of the inverted adventitia, eliminating the potential risk of thrombus formation. Furthermore, reinforcement of the intima by two adventitial layers, an external felt strip, and tube graft (Fig. 1C) lead to complete hemostasis, eliminating the need for hemostatic stitches and resulting in stable anastomosis. 

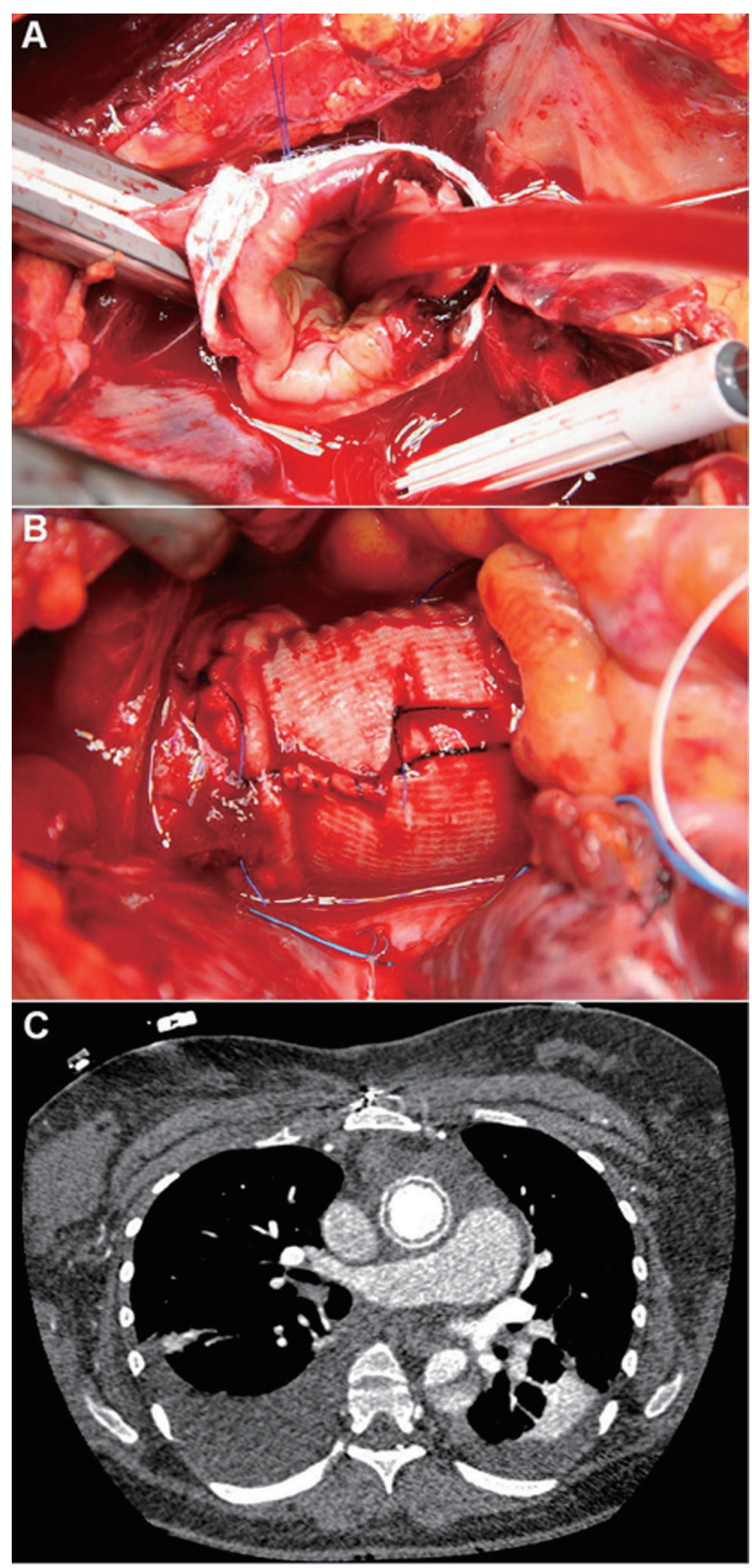

Fig. 2 Treatment of acute type A aortic dissection using adventitial inversion and telescopic graft insertion technique. (A, B) Intraoperative image showing the intima inverted into the aortic lumen with mattress sutures reinforced with an extraluminal circumferential felt strip on the distal anastomosis of the supracoronary tube graft in a 35-year-old female. (C) Early postoperative computed tomographic scans revealed no ridge projecting into the blood stream. Five days after surgery, the false lumen in the descending aorta was partially thrombosed.

\section{Conclusions}

We believe that this method is a worthwhile improvement in and optimization of the adventitial inversion technique for the distal anastomosis. We cannot, however, recommend this method for all aortic anastomoses in all circumstances. However, we suggest that this time-consuming technique is appropriate in patients presenting uncomplicated pathology requiring only supracoronary replacement of the ascending aorta.

\section{Disclosure Statement}

The authors have no financial or other interest in the manufacture or distribution of the devices and materials described above. The authors do not receive any financial incentives from the manufacturer of the devices and materials described above.

\section{References}

1) Floten HS, Ravichandran PS, Furnary AP, et al. Adventitial inversion technique in repair of aortic dissection. Ann Thorac Surg 1995; 59: 771-2.

2) Tanaka K, Morioka K, Li W, et al. Adventitial inversion technique without the aid of biologic glue or Teflon buttress for acute type A aortic dissection. Eur J Cardiothorac Surg 2005; 28: 864-9.

3) Kim SW, Sung K, Lee YT, et al. Aortic false lumen patency following the adventitial inversion technique for acute DeBakey Type I Aortic Dissection. J Card Surg 2010; 25: 548-53.

4) Heijmen RH, Gruendeman PF, Borst C. Intima-adventitia apposition in end-to-side arterial anastomosis: an experimental study in the pig. Ann Thorac Surg 1998; 65: 705-11.

5) Rylski B, Siepe M, Schoellhorn J, et al. An improved technique for aortic anastomosis: graft telescopic inversion. J Thorac Cardiovasc Surg 2010; 140: 934-5. 